\title{
Combination of Liver Resection with Methods of Local Thermal destruction in the Treatment of Metastases of Colorectal Cancer in the Liver
}

\author{
Chzhao Alexey V, Ionkin Dmitry A*, Olifir Anna A, Vishnevsky Vladimir A, Zhavoronkova Olga \\ I, Stepanova Yulia A, Karelskaya Natalia A, Zemskov Vladimir M and Kozlova Maria N \\ AV Vishnevsky National Medical Research Center of Surgery, Moscow, Russia
}

*Corresponding author: Ionkin Dmitry A, AV Vishnevsky National Medical Research Center of Surgery, Moscow, Russia.

To Cite This Article: Ionkin Dmitry A. Combination of Liver Resection with Methods of Local Thermal destruction in the Treatment of Metastases of Colorectal Cancer in the Liver. Am J Biomed Sci \& Res. 2019 - 4(4). AJBSR.MS.ID.000819. DOI: 10.34297/AJBSR.2019.04.000819

Received: August 03, 2019 | Published: August 12, 2019

\begin{abstract}
Relevance: Colorectal cancer (CRC) is one of the most common of the detection among malignant tumors. More than $80 \%$ of patients with metastases of the CRC to the liver are unsuitable for radical surgical treatment. It is this category of patients that shows the application of local thermal destruction methods.
\end{abstract}

The Aim: Improving the life quality and increase life expectancy (in combination of chemotherapeutic treatment) in patients with metastatic of colorectal cancer in the liver (CLM).

Materials and Methods: Cryo destruction have been performed in 24 patients with CLM. There were 11 women,13 men. The median age was

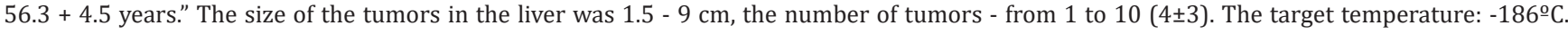
Exposure time: 3-5 min. Radiofrequency ablation (RFA) in a combination of cryo destruction was performed in 3 patients, Cryo + liver resection - in 7, a combination of liver resection + RFA + Cryo in 5 patients. Open intraoperative RFA and percutaneous RFA were performed in 20 patients, combination of liver resection and RFA in 16 patients. All patients subsequently underwent adjuvant chemotherapy, supplemented with regional chemoembolization in 9 cases.

Results: Life-threatening complications in postoperative period has been developed in 2 (8.4\%) patients: intra-abdominal bleeding in 1 (4.2\%) case, and multiple organ failure resulted in death on the 47th day after the intervention (liver resection+ Cryo+RFA) in $1(4.2 \%)$. Postoperative morbidity also includes biliary fistula in 2(8.3\%) patients, ascites- in 7(29.6\%), pleural effusion in 6(25\%), pneumonia - in 2(8.3\%). Complete pain and right upper abdominal discomfort reduction after the procedure were observed in $40 \%$, significant pain reduction - in $20 \%$ of patients. Median survival was $12.0 \pm 4$ months for Cryo only, 11,0 \pm 4 months for RFA only, 7,6 \pm 4 months for Cryo in combination of RFA, 13,5 \pm 5 months (p<0.05) for Cryo in combination with liver resection, $18,5 \pm 6$ months $(\mathrm{p}<0.05)$ for liver resection in combination with other methods of local destruction $(\mathrm{RFA}$, Cryo).

Conclusion: Combination of different methods of local thermal destruction in case of unresectable CLM improves the quality of life and with adjuvant chemotherapy could prolong survival rates.

Keywords: Local thermal destruction; Cryo destruction; RFA; Colorectal cancer liver metastases

\section{Introduction}

Colorectal cancer (CRC) is a common and lethal disease. CRC incidence and mortality rates vary markedly around the world. Globally, CRC is the third most commonly diagnosed cancer in males and the second in females, with 1.8 million new cases and almost 861,000 deaths in 2018 according to the World Health Organization GLOBOCAN database [1]. Current researches suggest that over 86 percent of patients diagnosed under the age of 50 are symptomatic at diagnosis, and despite this, they have a more advanced stage at diagnosis and poorer outcomes [2,3]. Liver metastases of
CRC are diagnosed synchronously with colorectal cancer in $25 \%$ of patients $[3,4]$. Over $23 \%$ of them also present extrahepatic metastases. Nearly a half of the remaining patients develop metachronous liver metastases within 3 years following bowel resection [3,4]. For patients with colorectal cancer liver metastases, liver resection is currently the only treatment with a curative intent and chance to prolong life $[3,5]$. Radical surgery is the "gold" standard in the treatment of metastatic tumors of the liver, allowing to achieve $37-45 \%$ 5-year survival, with a combined effect (surgical treatment and 
chemotherapy) - 50\%. The median overall survival in such patients is $35-40$ months [2].

Unfortunately, only $10-25 \%$ of patients with colorectal liver metastases are justified to radical surgical treatment [4]. Metastatic tumors in the remaining patients are considered unresectable, mainly due to their dissemination in both liver lobes, presence of extrahepatic metastases or the inability to preserve an adequate future liver remnant volume. Without specific treatment, the life expectancy of these patients is 5-11 months [4,5]. In such patients, palliative treatment is implemented, such as chemotherapy and thermal ablation of metastases. It is this category of patient's different method of local control could be applying such as cryoablation, radiofrequency, microwave and laser thermal ablation [6]. The development of cryosurgery techniques in the USSR in the 80s of the last centuries began with the study of cryotherapy in the liver and pancreas in an experiment that was later introduced into the clinic [7]. Cryo methods are widely used in the treatment of cancer [8]. Authors recommend the use of cryo destruction (cryoablation) as a palliative method for metastatic liver cancer in order to relieve pain, as well as to reduce the growth rate of neoplasms in locally advanced tumors, increase life expectancy (from 5-11 months with- out surgery to 14-18 months after surgical treatment) [9]. Cryo destruction not only leads to local destruction of the tumor, but also stimulates the immune response, improves the quality of life of patients $[7,9,11]$.

The first reports about the effect of radiofrequency energy on living tissues with their heating and the formation of coagulation necrosis belong to the last decade of the XIX century [4]. However, the practical application of RFA in the treatment of focal neoplasms of the liver has its history since the first half of the 90s of the 20th century [11]. In a multicenter randomized study for 15 years, the justification of the use of cryo destruction in patients with colorectal cancer metastases in the liver has been shown [4]. Long-term results were comparable with other methods of local destruction (Table 1). A large number of postoperative complications after cryoablation can be explained by the lack of a single protocol of the intervention, errors in the selection of patients and their management after surgery. Optimizing the tactics of surgical treatment in patients with metastatic colorectal cancer in the liver, the development of indications and contraindications for the use of local thermal destruction methods will reduce the morbidity and improve long-term results and also the quality of life of patients.

\begin{tabular}{|c|c|c|c|c|c|c|}
\hline \multirow{2}{*}{$\begin{array}{l}\text { Method of local } \\
\text { destruction }\end{array}$} & \multirow{2}{*}{ Local relapse (\%) } & \multirow{2}{*}{$\begin{array}{c}\text { Progression of oncological } \\
\text { disease (\%) }\end{array}$} & \multicolumn{3}{|c|}{ Survival } & \multirow{2}{*}{ Severe complications (\%) } \\
\hline & & & 1 year & 3 years & 5 years & \\
\hline Cryo & $12-39$ & $78-88$ & 84 & 37 & 17 & 29 \\
\hline MWA & $5-13$ & $50-78$ & 73 & 30 & 16 & 7 \\
\hline RFA & $10-31$ & $47-86$ & 85 & 36 & 24 & 6 \\
\hline
\end{tabular}

\section{Materials and Methods}

Since 2012 cryo destruction have been performed in 24 patients with CLM, RFA - in 23 patients. Indications for methods of local thermal destruction were:

a) Unresectable metastases of colorectal cancer in the liver

b) General contraindications for major surgical procedures (patients with severe somatic symptoms, coagulopathy, etc.).
Contraindications:

a) Lesion (close contact) of the loops of the intestine, the gallbladder, the walls of the stomach for a on a significant length.

b) Dissemination of the cancer process. In the pre- and postoperative period, all patients underwent clinical, laboratory, immunological, and instrumental examinations (Doppler ultrasound, computed tomography with contrast, and contrast-enhanced magnetic resonance imaging with DWI).

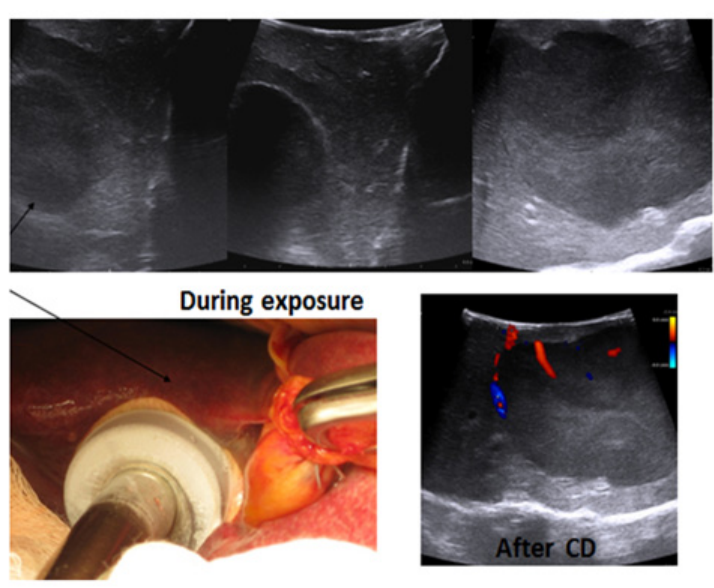

Figure 1: Intraoperative ultrasonography. 
There were 21 women, 26 men. The median age was $56.3+4.5$ years. The size of the tumors in the liver was $1.5-9 \mathrm{~cm}$, the number of tumors - from 1 to $10(4 \pm 3)$. The target temperature: $-186^{\circ} \mathrm{C}$. Exposure time: 3-5 min. During the operation, ultrasound monitoring of the effectiveness of the procedure was used (Figure 1). Radiofrequency ablation (RFA) in a combination of cryo destruction was performed in 3 patients, Cryo + liver resection - in 7, a combination of liver resection + RFA + Cryo - in 5 patients. Open intraoperative RFA and percutaneous RFA were performed in 20 patients, combination of liver resection and RFA in 16 patients.

All patients subsequently underwent adjuvant chemotherapy, supplemented with regional chemoembolization in 9 cases.

\section{Results}

Postoperative complications developed in 15 (37.5\%) of patients. According the classification of Clavien-Dindo, the complications we noted in stages can be divided as follows (Table 2).
Life-threatening complications were observed in 2 (8.4\%) patients. In the postoperative period intra-abdominal bleeding - 1(4.2\%) case; renal and hepatic failure led to the development of the syndrome of multiple organ failure and then to death on the 47 th day after the intervention (liver resection + Cryo + RFA) -1(4.2\%). Pain after the procedure: complete pain disappearance after the procedure observed in $40 \%$, significant pain reduction - in $20 \%$ of patients. Long-term results were $12.0 \pm 4$ months for Cryo only, 11,0 \pm 4 months for RFA only, 7,6 \pm 4 months for Cryo in combination of RFA, $13,5 \pm 5$ months $(\mathrm{p}<0.05)$ for Cryo in combination of liver resection, $18,5 \pm 6$ months $(\mathrm{p}<0.05)$ for liver resection in combination of other methods of local destruction (RFA, Cryo) (Figures $2 \& 3$ ). We have performed a retrospective study to assess the outcomes of patients receiving local thermal destruction - RFA, cryo destruction and combination for colorectal cancer metastases. We compared them against an age-sex matched group who had a similar treatment profile.

\begin{tabular}{|c|c|}
\hline \multicolumn{2}{|l|}{ Table 2: Postoperative morbidity. } \\
\hline Stage & Complication \\
\hline I stage & bscites (25\%); right-sided hydrothorax (16,6\%); right lower lobe pneumonia $(8,2 \%)$ \\
\hline II stage & $0 \%$ \\
\hline III stage & intra-abdominal bleeding (4,2\%) \\
\hline IV stage & Renal and hepatic failure led to the development of the syndrome of multiple organ failure and then to death on the 47 th day after \\
\hline V stage & the intervention (liver resection+ Cryo+RFA) $(4,2 \%)$.
\end{tabular}
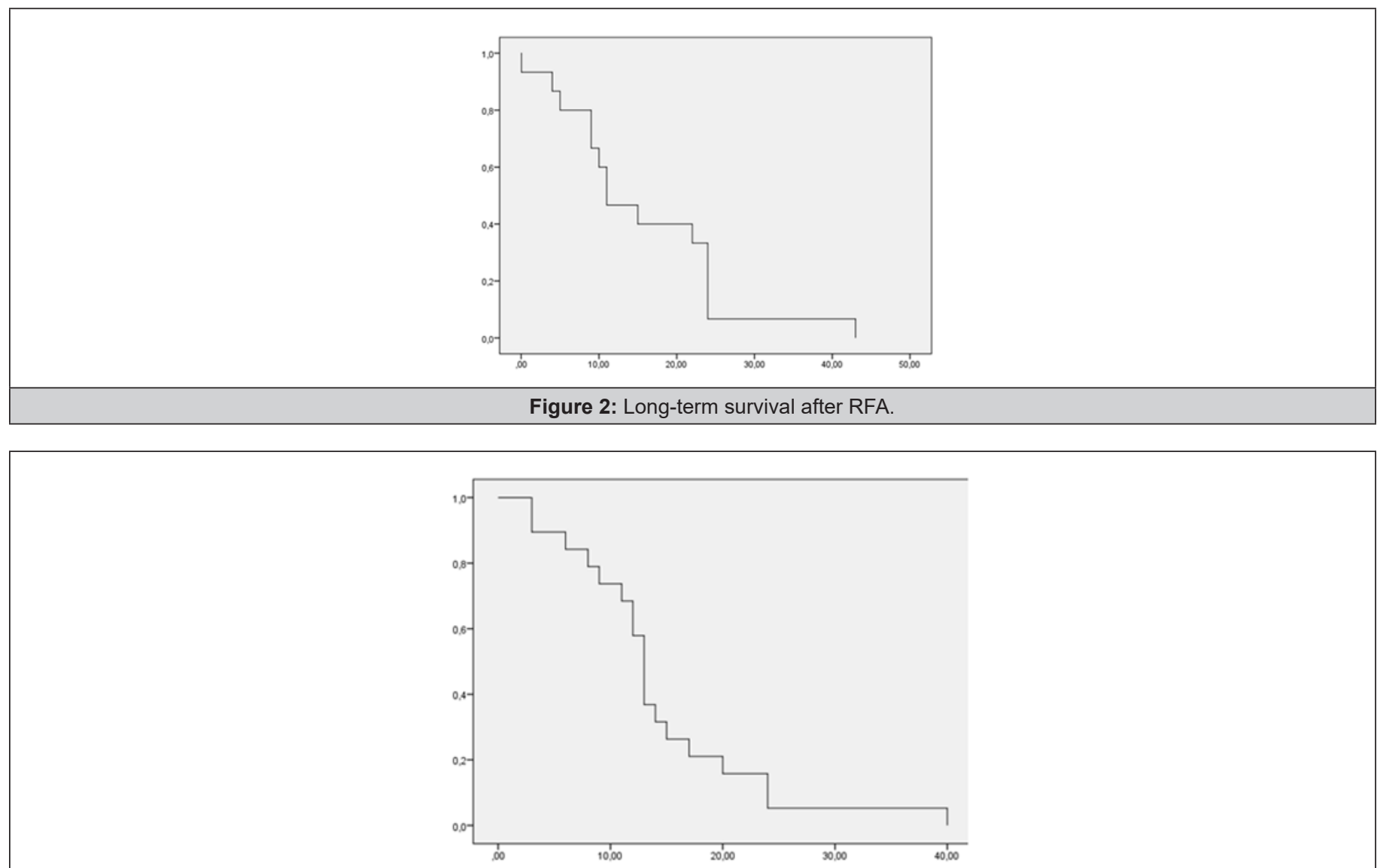

Figure 3: Long-term survival after Cryo. 
Colorectal cancer is considered a systemic disease. However, hepatic involvement is a crucial disease parameter, since liver metastases occur in more than $70 \%$ of patients and the disease course is largely determined by the extent of hepatic involvement (otherwise the residual functional hepatic reserve). Consequently, it is not surprising, that local therapy for macroscopic hepatic disease may extend beyond palliation for selected patients, resulting in delayed progression or death. Survival estimates were in favor of thermal destruction and suggested a marginal benefit. Ablation therapies have become increasingly popular due to their less invasive nature. Ablation modalities vary and include cryoablation, ethanol ablation laser ablation, RF ablation, MW ablation and irreversible electroporation. MW is the most recent evolution in ablation procedures and uses microwave energy for tumor destruction. In cases of colorectal hepatic metastases, "the rule of five" is usually followed (less than 5 lesions, less than $5 \mathrm{~cm}$ in diameter) [11] as an indication for ablation. Our results are remarkably consistent with previous studies that applied the same rule: Jones R. and Tang J reported a 5-years survival of 17, 16 and $23 \%$ [12] reported a median survival of 31 months in 122 patients. Our survival estimates after combination of Cryo, RFA and liver resection (18,6 months) were in line with these observations. Cryo destruction in connection with RFA, liver resection and adjuvant chemotherapy improve survival rate compared with RFA and Cryo only (11,0; 12,0 month). It is necessary to apply a multidisciplinary approach in the treatment of such patients

\section{Conclusion}

The technology and techniques used for ablation to treat liver metastases of CLM have continued to evolve in the past several decades and now include thermal methods, such as radiofrequency ablation and cryo destruction. Although it is commonly performed in conjunction with systemic therapy or other liver-directed treatment, ablation may offer a survival benefit for patients with metastatic disease in the liver. Methods of local thermal destruction in case of unresectable CLM could greatly improve the quality of life of patients, primarily by reducing pain. It is necessary to apply a multidisciplinary approach in the treatment of such patients. This is particularly true for small lesions not located adjacent to biliary or vascular structures. Cryo destruction in connection with RFA, liver resection and adjuvant chemotherapy lead to improve in survival rates. Further development of techniques to enhance ablation and study ablative methods as part of multimodality treatment may help patients achieve survival rates that approach those of surgical resection, the current treatment of choice.

\section{References}

1. Fitzmaurice C, Allen C (2017) Global, Regional, and National Cancer Incidence, Mortality, Years of Life Lost, Years Lived with Disability, and Disability-Adjusted Life-years for 32 Cancer Groups, 1990 to 2015: A Systematic Analysis for the Global Burden of Disease Study. JAMA Oncol 3(4): 524-528.

2. Cronin KA, Lake AJ, Scott S, Sherman RL, Noone AM, et al. (2018) Annual Report to the Nation on the Status of Cancer, part I: National cancer statistics. Cancer 124(13): 2785-2800.

3. Siegel RL, Miller KD, Jemal A (2019) Cancer statistics. CA Cancer J Clin 69(1): 7-34.

4. Jones R, Tang J, S Pathak (2011) Systematic review of ablation therapies for the treatment of unresectable colorectal liver metastases. HPB 13(2): 83-84.

5. Tombesi P, Vece FD, Sartori S (2015) Radiofrequency, microwave, and laser ablation of liver tumors: time to move toward a tailored ablation technique? Hepatoma Res 1: 52-57.

6. Chopra S, Dodd GD, Chintapalli KN, Leyendecker JR, Karahan OI, et al. (2001) Tumor recurrence after radiofrequency thermal ablation of hepatic tumors: spectrum of findings on dual-phase contrast-enhanced CT. AJR Am J Roentgenol 177(2): 381-387.

7. Alperovich BI (2001) Historical essay on cryosurgery in Russia. Cryosurgery in hepatology Achievements of cryomedicine. SPb Science 4-21.

8. Korpan NN (2001) Basics of cryosurgery. "Shringer-Verlag. Wien, New York, USA, pp. 325.

9. Revishvili A, Zhao AV (2019) Ionkin DA Cryosurgery. GEOTAR-Media, Moscow, Italy, pp. 376.

10. Xu KC, Niu LZ, Hu YZ (2007) Clinical experience of cryosurgery on 3580 patients with solid tumors. Technol Cancer Res Treat 6: 450-458.

11. Wong J, Cooper A (2016) Local ablation for solid tumor liver metastases: Techniques and treatment efficacy. Cancer Control 23(1): 30-35.

12. Veltri A, Sacchetto P, Tosetti I, Pagano E, Fava C, et al. (2008) Radiofrequency ablation of colorectal liver metastases: small size favorably predicts technique effectiveness and survival. Cardiovasc Intervent Radiol 31(5): 948-956 